\title{
Looking under the skin: the first steps in malarial infection and immunity
}

\section{Robert Ménard ${ }^{1}$, Joana Tavares ${ }^{1}$, Ian Cockburn², Miles Markus ${ }^{3}$, Fidel Zavala ${ }^{4}$ and Rogerio Amino'}

Abstract | Malaria, which is caused by Plasmodium spp., starts with an asymptomatic phase, during which sporozoites, the parasite form that is injected into the skin by a mosquito, develop into merozoites, the form that infects erythrocytes. This pre-erythrocytic phase is still the most enigmatic in the parasite life cycle, but has long been recognized as an attractive vaccination target. In this Review, we present what has been learned in recent years about the natural history of the pre-erythrocytic stages, mainly using intravital imaging in rodents. We also consider how this new knowledge is in turn changing our understanding of the immune response mounted by the host against the pre-erythrocytic forms.

Sterilizing immunity Immunity resulting in parasite clearance from the host

'Institut Pasteur, Unité de Biologie et Génétique du Paludisme, 28 Rue du Dr Roux, 75724 Paris Cedex 15, France.

${ }^{2}$ Department of Pathogens and Immunity, John Curtin School of Medical Research, Australian National University, Canberra, ACT 0200, Australia.

${ }^{3}$ School of Animal, Plant and Environmental Sciences, Faculty of Science, and Wits Research Institute for Malaria, Faculty of Health Sciences, University of the Witwatersrand, Private Bag 3 Wits, Johannesburg, 2050, South Africa.

4Johns Hopkins Malaria Research Institute, Bloomberg School of Public Health, Johns Hopkins University, 615 North Wolfe Street, Baltimore, Maryland 21210 , USA.

Correspondence to R.M. e-mail: robert.menard@pasteur.fr doi:10.1038/nrmicro3111 Corrected online 8 October 2013
Malaria is the most deadly parasitic infection of humans. Although economic development and the implementation of control measures during the twentieth century have eliminated malaria from many areas of the world ${ }^{1}$, the disease is still rampant in the tropics and in the poorest regions of the globe, affecting 3 billion people and killing up to 1 million annually ${ }^{2}$. Sub-Saharan Africa pays the heaviest toll, mainly because of the efficiency of Anopheles gambiae mosquitoes (which live in this region) as hosts of Plasmodium falciparum, the Plasmodium species that is most lethal to humans, delivering up to 120 infective bites per person per year ${ }^{3}$.

The symptoms of malaria are caused by cycles of parasite multiplication inside host erythrocytes, and various complications, including cerebral malaria, result from cytoadherence of infected erythrocytes to endothelia. However, infection with Plasmodium spp. starts when parasites are injected into the skin by a mosquito (FIG. 1). The first, pre-erythrocytic (PE) phase of infection is clinically silent. During this phase, the few parasites that are inoculated into the skin by the mosquito, called sporozoites, reach the liver. They invade and multiply inside hepatocytes into new parasite forms, termed merozoites, which invade erythrocytes. This preclinical parasite metamorphosis in the liver lasts $\sim 7-10$ days in humans and $\sim 2$ days in rodents.

The PE stages (namely, the sporozoite and ensuing liver stages) are present in very small numbers in the host. Therefore, they constitute a transmission bottleneck and are ideal vaccination targets, offering the possibility of preventing progression of the parasite life cycle before clinical illness occurs. PE forms have been the subject of intensive immunological research ever since the first demonstrations, in animal models and humans, that injection of attenuated parasites which do not cause blood infection confers protection against sporozoite challenge $e^{4-6}$. Today, this vaccination method is still the most efficient at offering sterilizing immunity against Plasmodium spp. infection.

This Review gives a perspective on our understanding of the PE phase of natural infection in rodents and of host defence against the PE stages in both rodents and humans. We begin by describing recent insights into PE infection. Many approaches have been used to better define sporozoite fate in the host, including powerful intravital imaging techniques that have been adapted for use in laboratory rodents (BOX 1). Although numerous molecular insights into the basic biology of the sporozoite and liver stages have also been gained, we restrict our coverage here to a few details relevant to our specific focus on infection at the organismal level. We then deal with the immunobiology of the PE stages, with emphasis on the immune response induced by live attenuated parasites (LAP). Most notable in recent years has been the increasing recognition of the importance of the initial skin step, which affects both infection by, and immunity to, PE parasites.

\section{An historical perspective on PE stages}

Defining the PE phase of malaria has been a long and winding road. After the discoveries of Plasmodium sp. parasites in the blood of a patient in 1880 by Laveran $^{7}$ and of the role of mosquitoes in Plasmodium spp. transmission in the late 1890s by Ross $^{8}$ and Grassi ${ }^{9}$, studies 


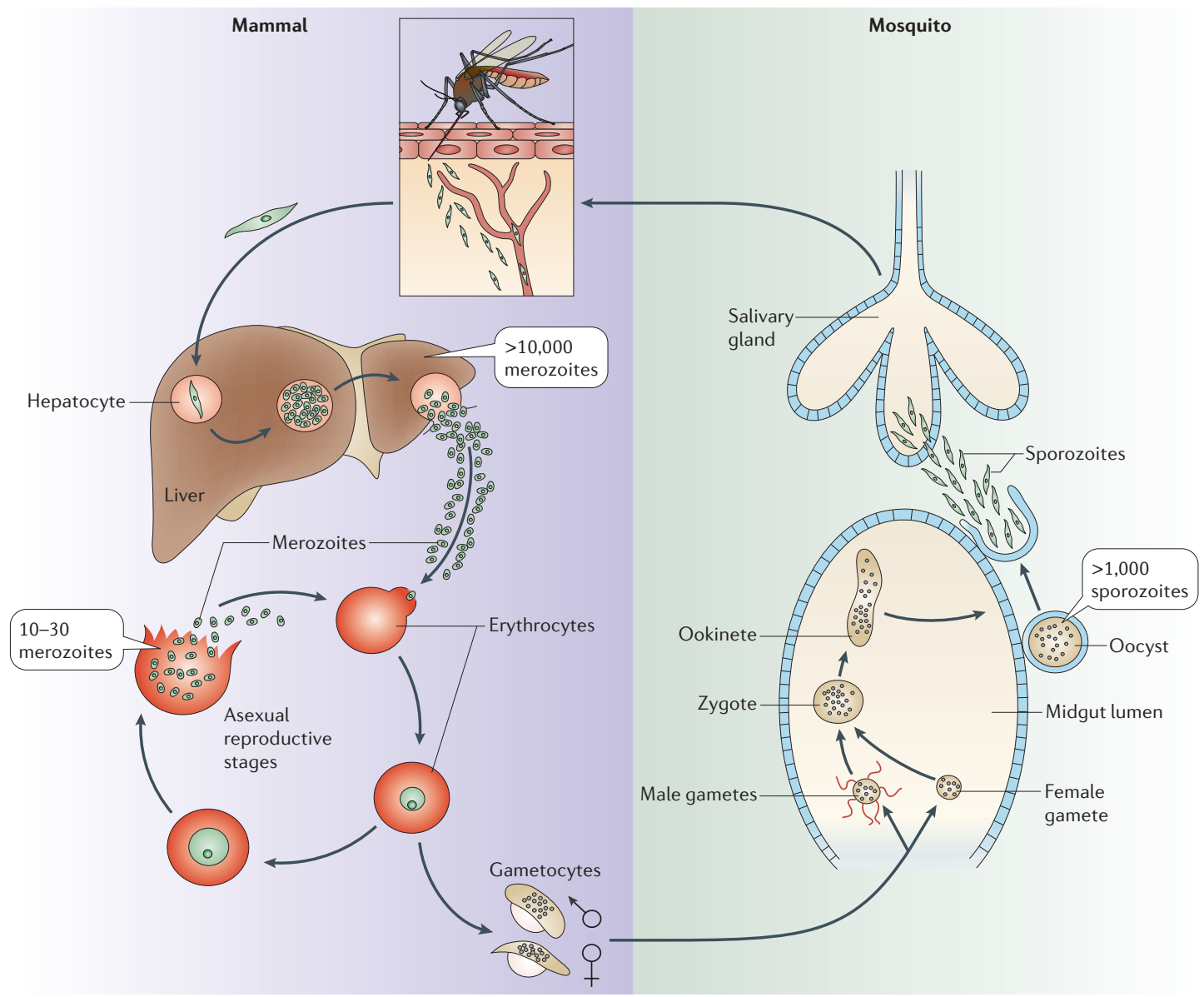

Figure 1 | The Plasmodium spp. life cycle. The life cycle of Plasmodium species that infect mammals. Symptoms of malaria are caused by cycles of parasite multiplication inside erythrocytes. One cycle is initiated as a result of erythrocyte invasion by a merozoite form and consists of $3-4$ nuclear divisions that generate $\sim 10-30$ new merozoites; a cycle typically lasts $\sim 24$ hours and $\sim 48-72$ hours in species that infect rodents and humans, respectively. Some intra-erythrocytic parasites transform into male or female gametocytes, which are taken up by a mosquito. Gametocytes egress from erythrocytes, activate into gametes and fuse in the mosquito midgut lumen. The motile zygote, called an ookinete, crosses the gut epithelium to transform into an oocyst, in which thousands of sporozoites develop. Sporozoites are released into the mosquito body cavity and later pass through salivary gland cells to enter the salivary ducts. After transmission into the skin of the mammalian host during a bite by the mosquito, motile sporozoites reach the liver, where they invade hepatocytes.

Schizonts

Multinucleate, intracellular parasite stages originating from a single organism that reproduces by schizogony. Schizonts contain many individual merozoites when mature.

\section{Hypnozoites}

Dormant, non-dividing, intrahepatocytic forms of certain Plasmodium species, including Plasmodium vivax and Plasmodium ovale, which infect humans.

\section{Recurrences}

New phases of parasite multiplication inside erythrocytes. These recurrences originate from intra-erythrocytic parasite forms (recrudescence) or hypnozoites (relapse). One intra-hepatocytic sporozoite generates tens of thousands of hepatic merozoites, which re-enter the bloodstream and invade erythrocytes. Numbers indicate parasite progeny after multiplication.

on PE stages in the first half of the twentieth century were mainly carried out using avian models of infection. The pioneering work of Raffaele and others in the 1930s revealed the presence of exo-erythrocytic schizonts in macrophages at the inoculation site in the skin, as well as in reticuloendothelial cells in the liver and spleen of infected birds, giving rise to successive PE cycles of infection ${ }^{10}$. In mammals, evidence for a PE 'tissue phase' was first obtained in 1948: Shortt and colleagues found that when sporozoites of the simian parasite Plasmodium cynomolgi were transmitted by mosquitoes to rhesus monkeys, these parasites developed as schizonts in parenchymal cells, but not other cells, in the liver ${ }^{11}$. Subsequent work showed that sporozoites of species that infect primates (including humans) undergo a single schizogonic cycle inside hepatocytes, although parasites of some species are thought to also persist inside hepatocytes for weeks to years as singly occurring PE forms called hypnozoites ${ }^{12,13}$. Recently, it has been speculated that at least some of the malarial recurrences which would conventionally be presumed to have a hypnozoite origin might actually have non-hypnozoite dormant stages as their source ${ }^{14}$.

The rodent-infecting Plasmodium species Plasmodium berghei was discovered in 1948 in Grammomys surdaster (an African tree rat) ${ }^{15}$, and in 1965, P. berghei sporozoites were shown to generate schizonts in the liver of laboratory rodents ${ }^{16,17}$. Since then, $P$. berghei and its sibling species Plasmodium yoelii, both of which are easily amenable to molecular genetic research ${ }^{18}$, have become practical and powerful models for investigating the basic biology of the PE stages. 


\section{Box 1 | Imaging approaches}

Recent advances in molecular biology and imaging technologies have allowed unprecedented opportunities to directly observe fluorescent and bioluminescent pathogenic microorganisms parasitizing their hosts in vivo and in real time.

Intravital fluorescence microscopy is an extremely powerful technique with which to study the very small numbers of parasites involved in the establishment of a pre-erythrocytic $(\mathrm{PE})$ infection, permitting both a qualitative and a quantitative view of parasite population behaviour at the tissue and cellular levels. The use of fast microscopes is required to track the highly motile sporozoites at the correct spatiotemporal resolution. Usually, individual parasites are recorded in a three-dimensional volume of $\sim 300 \times 300 \times 50 \mu \mathrm{m}^{3}$, using high-speed spinning-disk confocal microscopes. The use of high-speed two-photon microscopes with acousto-optical scanning technology can increase the depth of observation to $>500 \mu \mathrm{m}$.

By contrast, at least several thousand luciferase-expressing sporozoites are required for detection in the skin by bioluminescence imaging. However, despite the low temporal and spatial resolution of the technique, bioluminescence imaging is useful for locating parasite populations in the animal body and quantifying parasite behaviour (mainly development within host tissues), as one sporozoite can generate thousands of merozoites inside an infected cell.

Intravital fluorescence microscopy can also be used to gain molecular and functional insights into key steps of infection. This approach relies on the analysis of parasite behaviour using loss-of-function mutant parasites and/or intravital markers that act as reporters of various biological activities or processes (for example, quenched fluorescent substrates to analyse proteolysis, calcium indicators to analyse signalling, fluorescent reporters to analyse transcriptional activation, and propidium iodide to analyse cell traversal). The combination of the two provides powerful means to evaluate the role of molecules or activities in the parasite life cycle, as exemplified by the identification of cell traversal activity of sporozoites in the liver sinusoids.

\section{Gliding}

A substrate-dependent type of unicellular motility defined by the lack of cell deformation during movement.

Parasitophorous vacuole The vacuole inside which the parasite resides on host cell entry and throughout intracellular development.

Stellate cells Pericytes that are located between a hepatocyte and a sinusoidal endothelial cell; also called Ito cells.

\section{Infection by PE stages}

The skin as a branch point. Sporozoites can move at high speed by gliding motility ${ }^{19}$ and can traverse host cells by piercing host membranes ${ }^{20,21}$. They can also invade host cells inside a parasitophorous vacuole ${ }^{22,23}$, where they typically differentiate into merozoites. Around 100 sporozoites are injected by a mosquito in an experimental situation ${ }^{24,25}$, whereas in the wild, infected mosquitoes probably inoculate fewer than 50 sporozoites per bite on average ${ }^{26}$. In agreement with this, a limited parasite release rate through the mosquito proboscis ( 1-2.5 sporozoites per second) was reported by counting fluorescent $P$. berghei sporozoites during Anopheles stephensi salivation ${ }^{27}$ or transmission to mice ${ }^{28}$. It has long been considered that sporozoites are injected directly into blood vessels by mosquitoes, and the notion that sporozoites are instead inoculated into the extracellular matrix of the skin received experimental support only recently from experiments using bite site removal ${ }^{29}$ and interruption of mosquito feeding ${ }^{30}$. Intravital fluorescence microscopy (BOX 1) confirmed that most sporozoites are injected into the skin when the mosquito proboscis probes the skin for blood and ejects saliva $^{31}$. Sporozoites glide in the skin as fast as on glass slides ( 1-2 $\mu$ m per second in the first 30 minutes), display tortuous and apparently random three-dimensional movement patterns with no detectable tactism ${ }^{31-33}$ and can move for more than 1 hour, although their speed gradually decreases with time. Surprisingly, quantitative studies of $P$. berghei sporozoites injected by mosquitoes into the ear pinna of mice indicate that $\sim 25 \%$ and $\sim 15 \%$ of the sporozoites leave the skin by invading blood or lymphatic vessels, respectively, whereas $\sim 60 \%$ remain at the bite $\operatorname{site}^{32}$ (FIG. 2). Quantitative PCR experiments show a similar tripartite fate for P. yoelii sporozoites inoculated into the skin ${ }^{34}$. The exact proportions of parasites that follow each route is likely to be influenced by many factors, including the vessel density at the bite site and the parasite species.

Entering the liver. Most of the sporozoites injected into the bloodstream reach and remain in the liver ${ }^{35}$. Extensive biochemical work in the 1990s implicated circumsporozoite protein (CSP), the 'coat-forming' protein of the sporozoite ${ }^{36}$, in this process. CSP binds the particularly highly sulphated glycosaminoglycan chains in liver heparan sulphate proteoglycans (HSPGs) produced by hepatocytes and stellate cells ${ }^{37}$. Which domain of CSP, the amino-terminal domain ${ }^{38,39}$ or the carboxy-terminal thrombospondin type I repeat (TSR) domain ${ }^{40-42}$ (both of which display HSPG-binding capacity), is involved in CSP binding to liver HSPGs and, thus, in sporozoite homing to the liver has been a long-debated question. A recent genetic study showed that only the N terminus of CSP is exposed on free sporozoites, and the TSR is masked; cleavage of the $\mathrm{N}$ terminus was found to expose the TSR and trigger sporozoite invasion ${ }^{43}$. However, as intravenously injected sporozoites lacking the CSP N terminus infect the liver normally ${ }^{43}$, it is possible that neither the $\mathrm{N}$ terminus (dispensable) nor the TSR (masked) is important for sporozoite arrest in the liver.

Insights into the life of the sporozoite in the liver have come from studies of its aggressive cell traversal (CT) behaviour. Following the first description of sporozoite traversal of host cells ${ }^{20}$, which had been seen using macrophages in vitro, work focused on traversal of hepatocytes, which was observed both in vitro ${ }^{21}$ and in vivo ${ }^{21,44}$. A wild-type sporozoite traverses several hepatocytes before invading a final hepatocyte inside a parasitophorous vacuole, where it differentiates. Initial studies using wild-type sporozoites concluded that hepatocyte CT activates parasite invasion of hepatocytes ${ }^{45}$, as well as intracellular development ${ }^{46}$, via the activity of hepatocyte growth factor (HGF) released from the traversed cells. More recently, a 1.5-fold increase in the number of parasites developing inside cultured hepatocytes in the presence of excess HGF was observed for P. berghei but not for $P$. yoelii, leading to the conclusion that the role of HGF is crucial and species specific ${ }^{47}$. By contrast, studies using CT-deficient sporozoites paint a different picture, in which CT is important because it allows traversal of cell types other than hepatocytes ${ }^{48,52}$. CT-deficient sporozoites were initially shown to be highly impaired in liver infection in vivo, but to invade and develop inside hepatocytes normally in vitro ${ }^{48-50}$. The in vivo contributions of CT (FIC. 3) were later revealed by imaging of sporozoites lacking sporozoite protein essential for cell traversal 2 (SPECT2) ${ }^{49}$, a protein containing a membrane attack complex/perforin-like domain. CT allows sporozoite locomotion and access to blood capillaries in the skin, as $\sim 80 \%$ of SPECT2-null sporozoites 

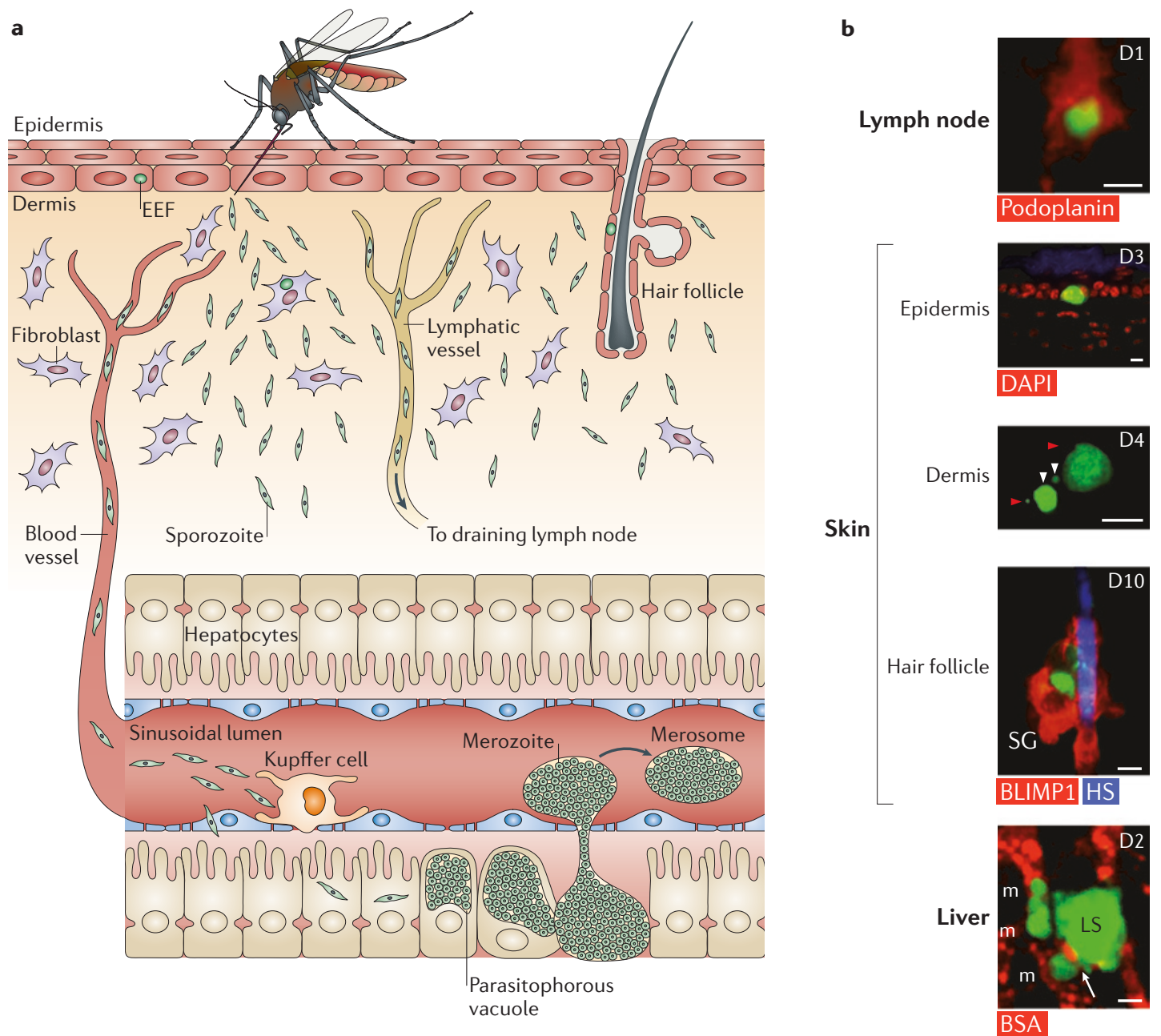

Figure 2 | The pre-erythrocytic phase in rodents. a | Motile sporozoites injected into the skin of rodents during a mosquito bite can leave the bite site via blood or lymphatic vessels, or stay in the skin. Sporozoites drained by lymphatic vessels can invade host cells in the proximal lymph node. Skin sporozoites can invade cells in the epidermis, dermis or associated with the hair follicle (exo-erythrocytic forms (EEFs)). Sporozoites that leave the bite site via the bloodstream stop in the liver, glide in the sinusoids (which are lined by endothelial cells and harbour Kupffer cells), wound Kupffer cells, cross the sinusoidal barrier, traverse several hepatocytes and invade a final hepatocyte within a parasitophorous vacuole. Merozoites, the erythrocyte-infecting parasite form, can be formed inside hepatocytes and skin cells. b |Confocal microscopy images from tissue cryosections (lymph node and epidermis) or intravital imaging (dermis, hair follicle and liver) show EEFs of the parasite (green). In the lymph node, the parasite is seen associated with a podoplanin-expressing cell. In the epidermis, the parasite is seen in the basal layer (cells stained with DAPI). In the dermis, an infected cell (right) releases merosomes (white arrowheads) and merozoites (red arrowheads). In a hair follicle of a Blimp1-GFP mouse, the parasite is shown associated with Blimp1-GFP-expressing cells in the sebaceous glands (SG); the hair shaft (HS) is visible because of autofluorescence. In the liver, a mature liver stage releases several merosomes $(\mathrm{m})$ into the blood circulation, indicated by bovine serum albumin (BSA); the sinusoidal barrier, through which the merosomes are released, is indicated (arrow). Days post-infection are shown; scale bars represent $10 \mu \mathrm{m}$. Lymph node image is reproduced, with permission, from REF. 32 @ (2006) Macmillan Publishers Ltd. All rights reserved. Dermis image is reproduced and and hair follicle image is modified, with permission, from REF. 72 @ (2010) National Academy of Sciences USA. Liver image is reproduced, with permission, from REF. 68 @ (2006) American Association for the Advancement of Science.

Kupffer cell

A resident macrophage in the liver; these cells mostly double-line the endothelial cell wall inside the sinusoid lumen. were rapidly immobilized in the $\operatorname{skin}^{51}$. CT then ensures sporozoite survival in the liver sinusoids, because $>90 \%$ of intravenously injected SPECT2-null sporozoites were rapidly phagocytosed by Kupffer cells ${ }^{52}$. Furthermore, CT facilitates sporozoite translocation across the liver sinusoidal barrier into the parenchyma, as $75 \%$ of the crossing events by wild-type sporozoites occurred by traversal of endothelial cells and/or Kupffer cells ${ }^{52}$. Intravital imaging showed that wild-type sporozoites use multiple pathways to cross the liver sinusoidal barrier ${ }^{52}$ (FIG. 3), contradicting the long-held view that sporozoites translocate across the barrier exclusively through Kupffer cells, a concept known as the gateway model ${ }^{44,53,54}$.

CT is therefore important for the progression of sporozoites through host tissues and for sporozoite resistance to host innate immunity en route to hepatocytes, but hepatocyte traversal does not seem to play a significant part in the establishment of infection. An 
a Skin
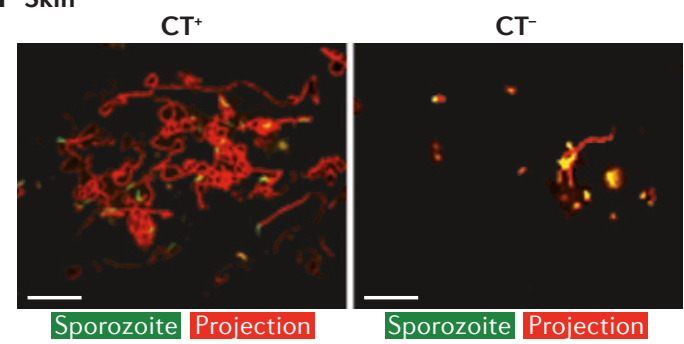

b Liver sinusoids
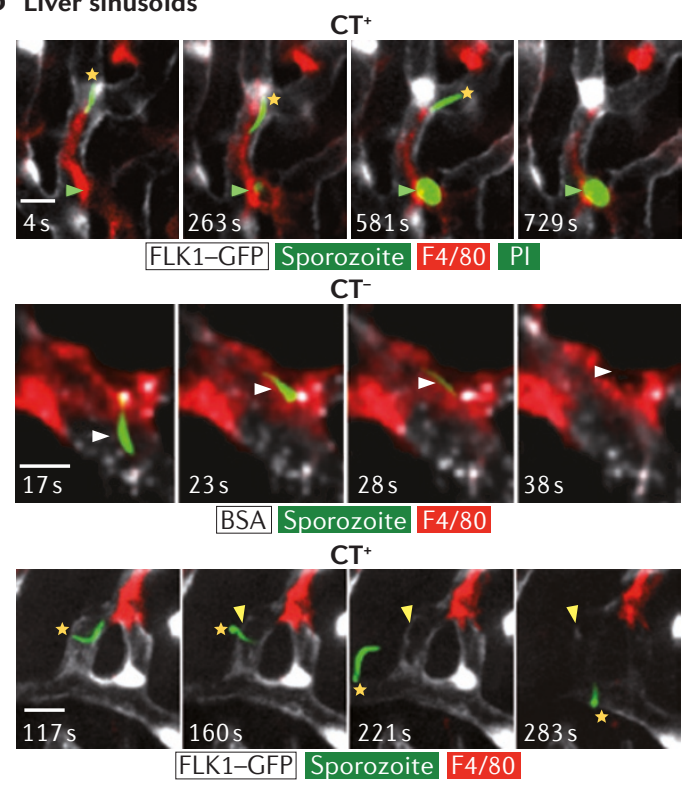

Figure $3 \mid$ Host cell traversal by sporozoites. Images obtained by intravital confocal imaging of cell traversal (CT) protein-expressing $\left(\mathrm{CT}^{+}\right)$or $\mathrm{CT}$-deficient $\left(\mathrm{CT}^{-}\right)$Plasmodium berghei sporozoites. a|CT in the skin. Maximal intensity projections of $\mathrm{CT}^{+}$(left) or $\mathrm{CT}^{-}$(right) sporozoite trajectories in the mouse ear over a 10-minute period. Immobilized $\mathrm{CT}^{-}$ sporozoites are shown in yellow. Scale bars represent $50 \mu \mathrm{m}$. b |CT in the liver sinusoids. Time-lapse microscopy of sporozoites (anterior tip indicated by a yellow star) in the liver of a mouse expressing fetal liver kinase fused to GFP (FLK1-GFP) (labelling endothelial cells) (upper and lower panels); Kupffer cells are labelled with F4/80-specific antibody; fluorescent bovine serum albumin (BSA) is used to label the liver sinusoids (middle panel). In the upper panel, a $\mathrm{CT}^{+}$sporozoite wounding a Kupffer cell is seen by the incorporation of propidium iodide (Pl; arrowheads) into the nucleus of the wounded Kupffer cell. In the middle panel, a $\mathrm{CT}^{-}$sporozoite durably interacts with, and is cleared by, a Kupffer cell (arrowheads). In the lower panel, a CT ${ }^{+}$ sporozoite extravasates (yellow arrowhead) by traversing an endothelial cell, visualized by fading of the fluorescence of the traversed endothelial cell (arrowheads). Times of imaging are shown. Scale bars represent $10 \mu \mathrm{m}$. Part b images are reproduced, with permission, from Ref. 52 (c) (2013) The Rockefeller University Press.

Apicoplast

A relict, non-photosynthetic plastid-like organelle of red-algal origin, inherited from secondary endosymbiosis. important question is how the sporozoite switches from CT to invasion. As mentioned above, cell invasion is associated with cleavage of CSP and exposure of the TSR domain ${ }^{43}$, possibly corresponding to cell contactdependent activation of sporozoite invasion. Turning off CT and membrane wounding, to avoid lysis of the parasitophorous vacuole membrane, is also obligatory for cell invasion, and indeed CT-deficient sporozoites readily invade cells ${ }^{51}$. A related question is, what triggers the switch? One study identified the particularly high levels of sulphation of liver HSPGs as the factor that induces the switch ${ }^{55}$. This conclusion, however, gives rise to the question of whether HSPGs can both cause arrest of sporozoites in the liver sinusoids ${ }^{40-42}$ and activate sporozoites to invade the final hepatocyte ${ }^{55}$. In addition, cleavage of glycosaminoglycan chains from HepG2 cell surfaces does not affect sporozoite invasion ${ }^{56}$. More work is needed to discover what triggers sporozoites to stop traversing cells, and how the switch to invasion operates.

Egressing from hepatocytes. Inside the parasitophorous vacuole, the parasite undergoes a spectacular development involving metamorphosis of the slender sporozoite into a large spherical liver stage, followed by schizogony to generate tens of thousands of merozoites in $\sim 2$ days ${ }^{57}$. Molecular insights have been gained into the composition of the parasitophorous vacuole membrane, the strategies for scavenging vital compounds from the host cell, and the contribution of the apicoplast, and these subjects have been reviewed elsewhere ${ }^{58-60}$. Another aspect of cellular parasitism that has been studied is the way the parasite controls host hepatocyte survival. Initially, host cell apoptosis is blocked ${ }^{61}$, possibly via inhibitor of cysteine proteases (ICP; called falstatin in $P$. falciparum $)^{62}$, which has been suggested to inhibit cathepsin L-like host cell proteases but not cathepsin B-type parasite proteases. The P. yoelii liver stage has been shown to modify the levels of several hepatocyte proteins, including $\mathrm{p} 53$, which is suppressed to enhance survival of the infected cell ${ }^{63}$. Later in development, the parasitophorous vacuole membrane is lysed $^{64}$, probably after activation of cysteine proteases belonging to the serine repeat antigen (SERA) family ${ }^{65}$, and the merozoites are released into the host cell cytoplasm. The parasite then controls a unique form of host cell death that has some features of apoptosis, such as mitochondrial disintegration and nuclear condensation, but lacks others, such as caspase activation; crucially, this type of cell death preserves the integrity of the host cell membrane ${ }^{66}$.

The final merozoite egress, which was long assumed to occur by mechanical bursting of the host cell ${ }^{67}$, is instead a sophisticated process of host cell membrane manipulation $^{68}$. Real-time in vivo imaging has shown that hundreds of merozoites are packaged together inside vesicles called merosomes, surrounded by the hepatocyte membrane (FIG. 2b). These structures bud off and detach from the cell into the sinusoidal lumen ${ }^{68,69}$ and have also been seen during P. falciparum infection in immunocompromised mice engrafted with human hepatocytes ${ }^{70}$. Merosomes are solid enough to pass through the heart and reach the lung circulation, where they have been observed liberating infectious merozoites ex $v_{i v o}{ }^{71}$. Merozoites manipulate the merosome membrane by impeding the exposure of phosphatidylserine 
on the outer leaflet ${ }^{64,68}$ (a normal 'eat-me' signal of dead cells), so merosomes are immunologically silent carriers. Therefore, whereas the sporozoite relies on its motile and membrane-wounding capacities to evade host phagocytes, the immotile merozoite has evolved a membranewrapping strategy.

A skin-draining lymph node step in PE malaria. The sporozoites that leave the skin inoculation site by invading lymphatic vessels remain in the proximal draining lymph node ${ }^{32}$. Although sporozoites are still motile on arrival in the subcapsular sinuses of the lymph node, most are quickly taken up by $\mathrm{CD} 11 \mathrm{c}^{+}$dendritic cells (DCs). However, a few parasites invade cells expressing podoplanin (a membrane protein found in lymphatic endothelial cells) (FIG. 2b), and the parasites in these cells do not complete exo-erythrocytic development ${ }^{33}$. The lymphatic route thus seems to be a dead end in terms of the parasite life cycle.

Surprisingly, the skin itself is a suitable niche for parasite development ${ }^{72,73}$. Intravital imaging of $P$. berghei sporozoites inoculated into the skin by mosquitoes revealed that up to $10 \%$ of the sporozoites develop in the dermis, the epidermis and in association with hair follicles ${ }^{72}$ (FIG. 2b). Although most skin parasites die before completing their intracellular development, both $P$. berghei and $P$. yoelii sporozoites can multiply to form merozoites inside skin cells (probably in dermal fibroblasts). Ex vivo experiments have shown that skin merozoites can infect erythrocytes $^{72}$. However, whether skin merozoites can initiate a blood infection in vivo could not be decisively demonstrated $\mathrm{d}^{72,73}$, although the observation of merosomes detaching from skin cells and moving within the $\operatorname{skin}^{72,73}$ favours this hypothesis. Sporozoites expressing CSP with a constitutively exposed TSR induce blood infection without leaving the $\mathrm{skin}^{43}$, a finding which further suggests that erythrocyte infection can be initiated by skin merozoites. Another striking revelation was the persistence of a small proportion of parasites for weeks in association with hair follicles ${ }^{72}$, an immunoprivileged site in the mammalian body ${ }^{74}$, raising the possibility that parasites associated with hair follicles are a source of infection recurrences in mammalian malaria.

The traditional view that Plasmodium spp. sporozoites travel only from the skin to the liver in mammals should thus be revisited. At least in rodents, after mosquito delivery, a large proportion of sporozoites ( $>50 \%$ in the ear) stay in the skin, where multiplication can occur, whereas other sporozoites end up in the draining lymph node until they are eventually cleared (FIG. 2). Whether the same picture holds for human malaria is still unknown. Nonetheless, the development of avian Plasmodium spp. in the skin ${ }^{75}$, the similarity between host cell invasion by $P$. yoelii and P. falciparum sporozoites $^{76}$, and an early description of Plasmodium vivax in a human lymph node ${ }^{77}$ all point to the possibility that a skin-draining lymph node component in the PE phase of the Plasmodium spp. life cycle is more conserved than previously appreciated.

\section{Protection against PE stages}

The PE stages are attractive targets for immunological intervention. During their journey to the liver, sporozoites are exposed to antibodies in tissue fluids and the bloodstream. Moreover, developing PE parasites in the skin and liver are the only parasite forms that are present in a nucleated cell and so capable of presenting antigens in association with major histocompatibility complex (MHC) class I molecules. There is, however, little evidence that natural exposure to parasites induces even partially protective immunity against the $\mathrm{PE}$ stages $^{78}$. By contrast, immunization with large doses of LAP, which are incapable of completing PE development and causing blood infection, has long been known to induce protection against sporozoite challenge in animal models ${ }^{4,5}$ as well as in humans ${ }^{6,79}$ (FIG. 4). Although LAP were initially radiation-attenuated sporozoites (RAS), it has been shown that parasites attenuated by genetic engineering ${ }^{80-91}$, drug treatment ${ }^{92-99}$ or chemical modification ${ }^{100}$ also act as potent vaccines in rodents and humans.

The basis of protection induced by RAS has been studied extensively in rodents. Protection efficiency seems to depend on the host-parasite combination, varying according to both the susceptibility of the host to infection and the ability of the host to mount effective immune responses ${ }^{101,102}$. Work in the 1980s first revealed an important role for CSP-specific antibodies, which immobilize sporozoites, in RAS-induced protection ${ }^{103,104}$. It was subsequently acknowledged that $\mathrm{CD} 8^{+} \mathrm{T}$ cells, which recognize parasite epitopes associated with $\mathrm{MHC}$ class I on the surface of infected hepatocytes, play a major part in killing infected hepatocytes ${ }^{105-108}$, although $\mathrm{CD}^{+} \mathrm{T}$ cells have also been implicated ${ }^{109,110}$. How these protective $\mathrm{CD}^{+} \mathrm{T}$ cells kill liver-stage parasites is still unclear. These cells use various cytotoxic effectors, such as perforin, FAS ligand, granzymes, interferon- $\gamma$ (IFN $\gamma)$ and TNF, which are expressed in response to inducible nitric oxide synthase (iNOS), but parasite-specific $\mathrm{CD}^{+}$ $\mathrm{T}$ cells lacking IFN $\gamma$, perforin and FAS ligand can still eliminate liver-stage parasites ${ }^{111,112}$. The recent direct observation of $\mathrm{CD}^{+} \mathrm{T}$ cells killing $P$. yoelii liver-stage parasites in vivo showed that parasite-specific $\mathrm{CD}^{+}$ $\mathrm{T}$ cells recruit both specific and nonspecific T cells to the infected hepatocyte, leading to multiple phenotypes of parasite death ${ }^{113}$. Therefore, liver-stage elimination seems to depend on the cooperative activity of multiple $\mathrm{CD}^{+} \mathrm{T}$ cells and probably also other cell types, and to involve diverse and functionally redundant cytotoxic effectors.

Historically, LAP have mainly served as models to guide the development of subunit vaccination approaches. However, the subunit vaccines developed so far have not matched the protective efficacy of LAP; this is also true for the most advanced vaccine, RTS,S, which is composed of B cell and T cell epitopes of $P$. falciparum CSP in hepatitis B virus-like particles ${ }^{114}$. The idea that protective immunity against $\mathrm{PE}$ stages would require the induction of immune responses to a broad range of parasite antigens has consequently become increasingly accepted, and it might be that only whole-parasite vaccines will 


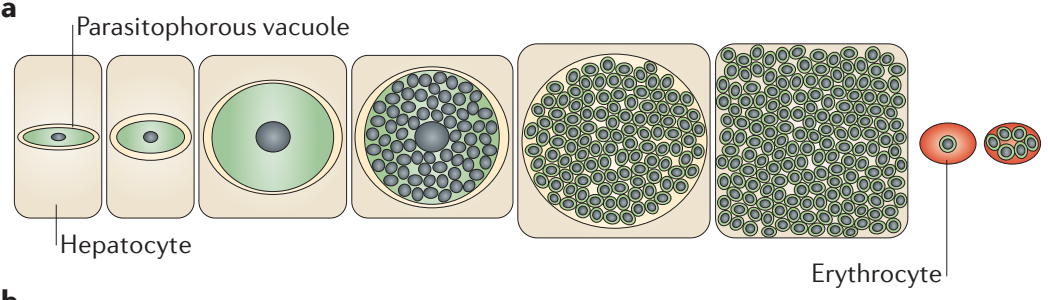

b

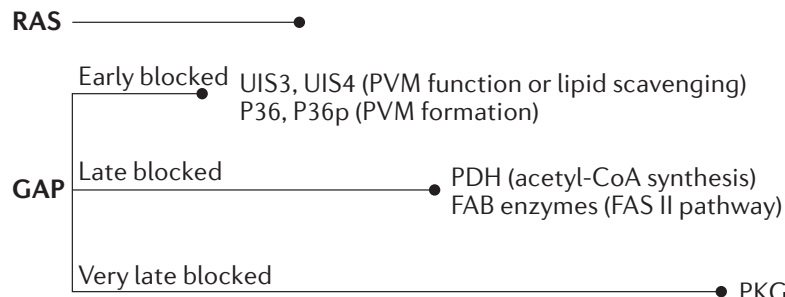

PKG (merozoite release)

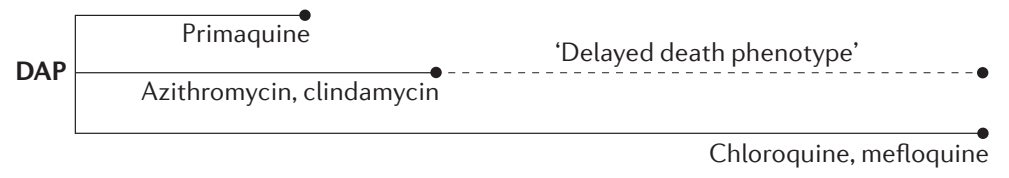

Figure 4 | Liver-stage maturation and live attenuated parasite-based vaccine strategies. a|Sporozoite transformation into merozoites inside a hepatocyte. Within a parasitophorous vacuole, the sporozoite transforms into a spherical liver stage that progressively enlarges and undergoes karyokinesis (involving the formation of up to 30,000 nuclei in $\sim 30$ hours) without cytokinesis, resulting in a multinucleate mother cell. Schizogony ends by the budding off of uninucleate daughter merozoites inside the vacuole. Merozoites egress from hepatocytes and invade erythrocytes. $\mathbf{b} \mid$ Stages of growth arrest of various live attenuated parasites (LAP). Growth of radiation-attenuated sporozoites (RAS) is arrested when DNA replication starts (at $~ 20-24$ hours after infection, midway through development), although depending on the irradiation dose, some nuclear division might continue. For genetically attenuated parasites (GAP), depletion of P36 and/or P36p (called P52 in some Plasmodium species) prevents the formation and/or maintenance of the parasitophorous vacuole membrane (PVM), causing very early liver-stage arrest, whereas depletion of UIS3 or UIS4 (which are lipid scavengers inserted in the PVM) causes rapid liver-stage elimination. Depletion of the apicoplast-localized pyruvate dehydrogenase ( $\mathrm{PDH}$; an enzyme that catalyses the conversion of pyruvate to acetyl-CoA) or FAB enzymes (type II fatty acid biosynthesis enzymes; that is, FABB/F, FABG, FABZ/A and FABI) causes mid-to-late liver-stage arrest, before merozoite formation and merozoite surface protein 1 (MSP1) expression. Conditional depletion of cyclic GMP-dependent protein kinase (PKG) in liver stages results in a very late liver-stage block, preventing the egress of fully formed merozoites. For drug-arrested parasites (DAP), the antifolate pyrimethamine and the 8-aminoquinoline primaquine both avert nuclear division of liver stages, causing mid liver-stage arrest. Azithromycin and clindamycin, which are macrolide and lincosamide antibiotics, respectively, inhibit apicoplast biogenesis in the liver stage; development is not arrested, but gives rise to non-infectious merozoites, blocked midway during their intra-erythrocytic growth (called the 'delayed death phenotype'). Chloroquine, a 4-aminoquinoline, and mefloquine, a 4-methanolquinoline, do not affect liver-stage growth and specifically inhibit haemozoin formation in infected erythrocytes, preventing the growth of intra-erythrocytic asexual parasites. exposed to just 15 infectious mosquito bites on each of three occasions and kept under chloroquine cover (see below) were protected for a prolonged period ${ }^{98,99}$. This compares somewhat favourably with RTS,S, which gives 32-85\% protection under similar laboratory challenge conditions ${ }^{118-120}$. No LAP have yet been tested under stringent field conditions, whereas RTS,S has been reproducibly shown to confer protection against clinical disease, although it has not exhibited any ability to block infection by parasites ${ }^{121-123}$.

Protective forms of LAP. A crucial issue is to determine which forms of the parasite are responsible for inducing LAP-mediated protection. Early evidence indicated a role for the liver stages rather than sporozoites, when it was discovered that RAS invade hepatocytes normally but stop developing at the onset of nuclear division ${ }^{124}$, roughly midway through liver-stage development (FIG. 4). Reports that protection depends on the sporozoite irradiation dose $\mathrm{e}^{125,126}$, with the most protective dose allowing several nuclear divisions, further suggested that late arrest of liver-stage maturation confers better protection than earlier arrest. LAP models based on drugarrested parasites (DAP) recently confirmed this idea. Intravenous injection of normal sporozoites into mice treated with chloroquine $e^{92-94}$ or mefloquine ${ }^{95}$, which have no effect on parasites in the liver but kill erythrocytic stages, can protect the host; compared with the number of RAS required for protection, fewer of these fully developing DAP are required for protection against subsequent challenge. Similar results have been obtained for treatment with clindamycin or azithromycin ${ }^{96,97}$, which interfere with the development of liver-stage parasites and render hepatic merozoites non-infective. As mentioned above, protection can be induced by just 45 immunizing mosquito bites delivering infectious sporozoites to humans under chloroquine cover ${ }^{98,99}$, whereas $>1,000$ bites delivering RAS alone were required for protection ${ }^{79}$. Remarkably, protection in normal sporozoite-treated volunteers is long-lasting, with four out of six treated volunteers still protected after 2 years, and this protection seems to be correlated with parasitespecific, multifunctional T effector memory cells ${ }^{99}$. Although after immunization with wild-type sporozoites under chloroquine cover the parasites eventually reach erythrocytes, which might induce blood-stage immunity ${ }^{127}$, it was recently shown that this DAP model essentially confers PE immunity, as it protects against sporozoite, but not blood-stage, challenge ${ }^{128}$.

The direct demonstration that late-blocked liver stages induce better protection than RAS in the absence of any blood-stage contribution came from studies on genetically attenuated parasites (GAP) in mice (FIG. 4). The first-generation GAP lack genes crucial to the biogenesis or maintenance of the parasitophorous vacuole membrane ${ }^{80-86}$. These parasites are blocked soon after sporozoite invasion and provide protection similar to or lower than that conferred by RAS. The discovery of the essential role of the apicoplast-resident fatty acid synthesis type II (FAS II)dependent pathway during late liver-stage development ${ }^{129}$ inspired the creation of the second-generation GAP, 
which are deficient in the FAS II pathway ${ }^{87-90}$. P. yoelii GAP lacking FABB/F, an enzyme involved in the FAS II pathway, are blocked later than RAS but before merozoite formation ${ }^{90}$. After intravenous immunization, they induce broader and more protective $\mathrm{CD} 8^{+} \mathrm{T}$ cell responses than RAS, involving a larger fraction of effector memory $\mathrm{CD}^{+} \mathrm{T}$ cells, as well as some degree of cross-stage protection against challenge with blood-stage parasites ${ }^{90}$. This superior protection could be due to the expression of antigens specific to late liver-stage parasites, directing stronger anti-liver-stage protection, or due to the expression of antigens shared with erythrocytic merozoites, potentially providing protection against both blood-stage and liver-stage parasites (FIG. 4). A further block in GAP development has been achieved by disruption of the cyclic GMP-dependent protein kinase, specifically preventing merozoite release from the infected hepatocyte ${ }^{91}$. Given that liver-stage development follows large transcriptomic changes in the parasite, GAP arrested at different developmental stages express different and unique sets of genes; for example, $\sim 480$ genes are co-expressed by sporozoites and merozoites ${ }^{130}$, whereas $\sim 770$ transcripts are differentially expressed in mid ( 24 hour) versus mid-to-late (40-50 hour) P. yoelii liver-stage infection ${ }^{131}$ (schematically corresponding to RAS and the FABB/F-null GAP, respectively).

LAP delivery into the skin. Studies involving late-arresting parasites have highlighted a role for late liver-stage antigens in protection after intravenous injection of LAP; however, work using RAS has shown that immune responses following inoculation into the skin are largely induced in skin-draining lymph nodes ${ }^{132,133}$. The development of a protective $\mathrm{CD}^{+} \mathrm{T}$ cell response after RAS delivery into the skin was dissected using transgenic $\mathrm{CD} 8^{+}$ T cells specific for an epitope of $P$. yoelii CSP (CSP-Tg) $)^{134}$. After RAS injection into the ear via syringe or mosquito, CSP-Tg CD8 ${ }^{+} \mathrm{T}$ cells are primed in the draining lymph node of the ear and, to a lesser extent, in the spleen, before migrating to the liver and other lymphoid organs, but are minimally activated in the draining lymph nodes of the liver ${ }^{132}$. Moreover, surgical ablation of the lymph node draining the injection site drastically decreases the number of activated $\mathrm{CD}^{+} \mathrm{T}$ cells in the liver ${ }^{132}$. It was also shown that in the draining lymph node, sporozoite antigen is cross-presented by DCs to naive CD8 ${ }^{+} \mathrm{T}$ cells to induce the protective $\mathrm{T}$ cell response ${ }^{135,136}$. Interestingly, antigen continues to be presented for up to 2 months following immunization via the skin with RAS or GAP, and this persistent antigen presentation is crucial for the complete development and expansion of protective CD8 ${ }^{+}$ T cell responses ${ }^{90,137}$.

Which parasite forms induce protective responses in the skin-draining lymph node remains unclear. Because dead (heat-inactivated) sporozoites left in the skin are not protective ${ }^{138}$, the protective material might be sporozoites that actively reach the draining lymph node of the skin ${ }^{32}$ or those that develop inside skin cells ${ }^{72}$, and proteins that are continuously shed by gliding and cell-traversing sporozoites in the skin and/or draining lymph node ${ }^{51}$ might also play a part. Importantly, if the immunogenic forms are those sporozoites that reach the draining lymph node and develop there to only a limited extent ${ }^{32}$, then late-blocked GAP might not be more protective than early-blocked GAP when injected into the skin.

Another question concerns the protective efficacy of LAP injection into the skin. As mentioned above, all LAP studies carried out so far in humans have delivered the LAP to the skin via mosquitoes ${ }^{79}$. One exception has been the use of cryopreserved RAS, which did not protect after intradermal injection ${ }^{116}$, but provided protection after intravenous injection ${ }^{139}$. Recent work in rodents has yielded contradictory results. One study reported lower protective efficacy after intradermal compared with intravenous immunization with $P$. berghei RAS or $\mathrm{DAP}^{140}$. However, other studies using $P$. yoelii $\mathrm{DAP}^{95}$ and $\mathrm{RAS}^{141}$ have recorded similar protection levels for intradermal and intravenous immunization. Some researchers who used P. yoelii ${ }^{95}$ or P. berghei $i^{140,142}$ have found the parasite load in the liver to be lower after intradermal versus intravenous injection of RAS, suggesting that protective efficacy is related to the ability of sporozoites to reach the liver ${ }^{116,34}$. By contrast, others using P. yoelii sporozoites have noted that the parasite load in the liver does not depend on the injection route ${ }^{34}$.

These discrepancies are likely to be due, at least in part, to different intradermal injection procedures and to the volumes used, as sporozoites display reduced

\section{Box 2 | Immunobiology of Plasmodium spp. pre-erythrocytic stages: outstanding questions}

- What are the parasite and host molecules that mediate sporozoite homing to, and arrest in, the liver, and can these steps in the life cycle be inhibited by antibodies?

- How do sporozoites coordinate motility, cell traversal and intravacuolar cell invasion?

-What is the basis of sporozoite transformation into a hypnozoite, does the process occur in cells other than hepatocytes, and can hypnozoite-containing cells be eliminated by leukocytes?

- Do human-infecting Plasmodium spp. develop in the skin?

- Can injection of live attenuated parasites (LAP) into the skin be used as a mass vaccination method for humans?

- Which parasite forms trigger immunity after injection of LAP into the skin?

- How are parasitized hepatocytes killed by host cells?

- Which T cell subset is most efficient at killing infected hepatocytes in humans?

-What are the relative contributions of antibodies and T cells for protection in humans?

- How can new protective pre-erythrocytic-stage antigens be identified to fuel the development of subunit vaccines? 


\section{Box 3 | Live attenuated parasites as vaccines for humans}

Between 1972 and 1999, studies involving a total of $\sim 30$ volunteers showed that an average of 1,000 bites by irradiated Plasmodium falciparum-infected mosquitoes, divided into 8-10 sessions over the course of an 10-month immunization schedule, resulted in protection in $90 \%$ of individuals on exposure to the bites of five infected mosquitoes $\sim 10$ weeks after the last immunization, and for up to 10 months when secondary immunizations were carried out ${ }^{79}$. Recently, volunteers were also found to be protected by drug-arrested parasites (DAP) — that is, normal sporozoites delivered under chloroquine cover by mosquitoes ${ }^{98,99}$. The company Sanaria Inc. was created in 2003 to manufacture cryopreserved $P$. falciparum radiation-attenuated sporozoites (RAS) for human vaccination against malaria ${ }^{115}$.

\section{Efficiency}

Experimental evidence suggests that live attenuated parasite (LAP)-mediated protection is directed at multiple antigens and could therefore be strain transcendent, which would allow host genetic restriction to be overcome. Strain transcendence remains to be demonstrated, as all challenges in human studies to date have used the same P. falciparum strain as the immunizing strain.

\section{Administration route}

Historically, LAP vaccination in humans has been achieved only via mosquito bites, which cannot be envisaged as a reliable mass-vaccination system. Sporozoites must therefore be injected by syringe via a clinically approved administration route (subcutaneous, intradermal or intramuscular); the intravenous route is least likely to be approved for mass vaccination. However, protection of humans has not yet been demonstrated by non-mosquito delivery of LAP in skin or muscle. Recently, it was found that five intravenous injections of $>10^{5}$ cryopreserved RAS protected all six tested patients against a challenge by bites of five mosquitoes 3 weeks after the last immunization dose, whereas four intravenous immunizations protected only six of nine patients ${ }^{139}$.

\section{Safety}

LAP doses must be devoid of both 'breakthrough' infection-causing parasites ( $100 \%$ attenuation) — an achievable goal for genetically attenuated parasites (GAP) — and viral and bacterial contaminants after parasite cultivation in human erythrocytes and mosquitoes.

\section{Large-scale use}

Sporozoites are generated only in mosquitoes, and their collection requires microdissection of mosquito salivary glands, a procedure that cannot easily be scaled up to the levels required for immunization in the field. A mosquito-free, tissue culture-based sporozoite production system would greatly boost the prospects of producing LAP for use in humans, but attempts have so far yielded only non-infectious sporozoites. Currently, live sporozoites can be stored only by cryopreservation at ultra-low temperature in liquid nitrogen, a process that kills most sporozoites, at least those of Plasmodium yoelii. Therefore, at the present time, LAP vaccines can be envisaged only for restricted human populations in the developed world, such as military personnel and travellers. proteins which are secreted across the parasitophorous vacuole membrane by developing parasites might be crucial protective antigens, and proteins that are deposited by traversing sporozoites might also be presented by cells that recover from the traversal event. Mechanisms of protein export across the parasitophorous vacuole membrane in the infected erythrocyte are becoming increasingly well defined ${ }^{144,145}$. By contrast, protein secretion in the hepatocyte cytosol has received less attention. CSP bears a so-called PEXEL motif, which is present in many exported proteins in the erythrocyte; however, although this motif was found to be important for CSP translocation into the hepatocyte cytosol ${ }^{146}$, it has no effect on the ability of CSP-specific CD8 ${ }^{+} \mathrm{T}$ cells to recognize and kill parasites ${ }^{136}$. The basis of liver-stage protein translocation into the hepatocyte cytosol and entry into the MHC class I presentation pathway are clearly important areas for future investigation.

\section{Conclusions and perspectives}

Recent research into the in vivo fate of Plasmodium spp. sporozoites in mice has changed our understanding of the PE phase of mammalian malaria. In mice, the skin injection site and its draining lymph node, where the majority of parasites remain, are integral components of the PE phase and the site of the first parasite encounter with the host immune system. The most pressing question is whether the same tripartite picture of the initial PE phase - in which sporozoites can remain in the skin or exit via the draining lymph node or the bloodstream - holds true in humans. There are many unknown factors concerning sporozoite infection in the rodent host, including the molecular basis of the tripartite fate of sporozoites inoculated into the skin and of sporozoite retention in the liver, the functional importance (if any) of epidermal and hair follicle-associated parasite subpopulations, and the fate and possible subversion of traversed host cells.

Much remains to be done if we are to understand the host responses that are generated by injection of sporozoites into the skin (BOX 2). Research in this area will be facilitated by the experimental accessibility of the skin and draining lymph nodes, particularly in respect of the powerful immuno-imaging approaches that are now available, as well as by recent progress in understanding skin immunity ${ }^{147}$. The initial aims are the identification of the host cell subset (or subsets) involved in antigen capture and in priming the protective $\mathrm{CD}^{+} \mathrm{T}$ cells in the draining lymph node, as well as the determination of which LAP forms, whether actively reaching the draining lymph node or remaining in the skin, cause protection. It will also be important to investigate the interaction with, and potential subversion of, regulatory $\mathrm{T}$ cells. These cells are abundant in the epidermis of human skin, and sporozoites glide extensively in the epidermis in rodents. A recent meta-analysis of $>1,900$ immunization studies led to the prediction that live sporozoites in the skin induce the expansion of antigenspecific regulatory $\mathrm{T}$ cells that ensure re-infection and systematically deactivate, rather than boost, vaccineinduced immune responses to skin-derived antigens ${ }^{148}$. 
The ultimate question is whether a PE vaccine could be deployed on a large scale. LAP approaches are efficient, but their implementation still requires major technical and logistical hurdles to be overcome ${ }^{149}$ (BOX 3). Regardless, LAP-based studies remain valuable and might lead to the identification of specific sets of protective antigens. In the meantime, efforts to develop subunit vaccines, primarily virus vectored, have yielded promising results ${ }^{150}$. For example, immunization against epitopes from two sporozoite proteins using prime-boost strategies based on adenovirus and modified vaccinia Ankara (MVA) virus confers potent protection and elicits a prolonged effector memory response in the liver ${ }^{151}$. Until now, subunit vaccinology has focused on just a few antigens, and mainly CSP. Although CSP is clearly a major protective antigen following RAS vaccination ${ }^{152}$, it is also obvious that $\mathrm{CD}^{+} \mathrm{T}$ cell-mediated protection can be mounted independently of $\mathrm{CSP}^{153}$. An important priority for future research is therefore to identify new protective $\mathrm{T}$ cell antigens that possibly elicit greater protection than CSP. The recent technical breakthroughs in the malaria research field, from genome sequencing to cellular imaging, now make it possible to envisage systematic approaches towards achieving this goal ${ }^{154}$.
1. Carter, R. \& Mendis, K. N. Evolutionary and historical aspects of the burden of malaria. Clin. Microbiol. Rev. 15, 564-594 (2002).

2. WHO. Malaria fact sheet. WHO Media Center [online], http://www.who.int/mediacentre/factsheets/fs094/en/ index.html (2013).

3. Mbogo, C. M. et al. Spatial and temporal heterogeneity of Anopheles mosquitoes and Plasmodium falciparum transmission along the Kenyan coast. Am. J. Trop. Med. Hyg. 68, 734-742 (2003).

4. Mulligan, H. W., Russell, P. F. \& Mohan, B. N. Active immunization of fowls against Plasmodium gallinaceum by injections of killed homologous sporozoites. J. Malaria Inst. India 4, 25-34 (1941)

5. Nussenzweig, R. S., Vanderberg, J., Most, H. \& Orton, C. Protective immunity produced by the injection of X-irradiated sporozoites of Plasmodium berghei. Nature 216, 160-162 (1967). The first vaccination of mice against sporozoite challenge.

6. Clyde, D. F., Most, H., McCarthy, V. C. \& Vanderberg, J. P. Immunization of man against sporozoite-induced falciparum malaria. Am. J. Med. Sci. 266, 169-177 (1973).

The first vaccination of humans against malaria.

7. Laveran, A. Un nouveau parasite trouvé dans le sang des malades atteints de fièvre palustre: origine parasitaire des accidents de l'impaludisme. Bull. Mem Soc. Med. Hop. Paris 17, 158-164 (in French) (1881).

8. Ross, R. On some peculiar pigmented cells found in two mosquitos fed on malaria blood. Br. Med. J. 2 , 1786-1788 (1897).

9. Grassi, B., Bignami, A. \& Bastianelli, G. Ulteriori ricerche sul ciclo dei parassiti malarici umani nel corpo del zanzarone. Atti R. Accad. Lincei 8, 21-28 (1899) (in Italian).

10. Huff, C. G. Life cycle of malarial parasites. Annu. Rev. Microbiol. 1, 43-60 (1947).

11. Shortt, H. E., Garnham, P. C. C. \& Malamos, B. The pre-erythrocytic stage of mammalian malaria. Br. Med. J. 1, 192-194 (1948).

The first demonstration that sporozoites of Plasmodium spp. which infect mammals transform in the liver.

12. Krotoski, W. A. et al. Demonstration of hypnozoites in sporozoite-transmitted Plasmodium vivax infection. Am. J. Trop. Med. Hyg. 31, 1291-1293 (1982).

13. Markus, M. B. The hypnozoite concept, with particula reference to malaria. Parasitol. Res. 108, 247-252 (2011).

14. Markus, M. B. Dormancy in mammalian malaria. Trends Parasitol. 28, 39-45 (2012).

15. Vincke, I. H. \& Lips, M. Un nouveau Plasmodium d'un rongeur sauvage du Congo: Plasmodium berghei n. $\mathrm{sp}$. Ann. Soc. Belge Med. Trop. 28, 97-104 (1948) (in French)

16. Yoeli, M., Most, H. \& Boné, G. Plasmodium berghei cyclical transmissions by experimentally infected Anopheles quadrimaculatus. Science 144, 1580-1581 (1964).

17. Yoeli, M., Vanderberg, J., Upmanis, R. S. \& Most, H. Primary tissue phase of Plasmodium berghei in different experimental hosts. Nature 208, 903 (1965).

The first observation of $P$. berghei schizonts in the liver of rodents.

18. van Dijk, M. R., Janse, C. J. \& Waters, A. P. Expression of a Plasmodium gene introduced into subtelomeric regions of Plasmodium berghei chromosomes. Science 271, 662-665 (1996).

The first genetic transformation by homologous recombination in $P$. berghei erythrocytic stages.

19. Vanderberg, J. P. Studies on the motility of Plasmodium sporozoites. J. Protozool. 21, 527-537 (1974).

20. Vanderberg, J. P., Chew, S. \& Stewart, M. J.

Plasmodium sporozoite interactions with macrophages in vitro: a videomicroscopic analysis. J. Protozool. 37 528-536 (1990)

The first observation of Plasmodium sp.sporozoites traversing host cells in vitro.

21. Mota, M. M. et al. Migration of Plasmodium sporozoites through cells before infection. Science 291, 141-144 (2001)

A study that shows the membrane-wounding activity of Plasmodium spp. sporozoites.

22. Hollingdale, M. R., Leef, J. L., McCullough, M. \& Beaudoin, R. L. In vitro cultivation of the exoerythrocytic stage of Plasmodium berghei from sporozoites. Science 213, 1021-1022 (1981).

23. Mazier, D. et al. Complete development of hepatic stages of Plasmodium falciparum in vitro. Science $227,440-442$ (1985).

24. Ponnudurai, T., Lensen, A. H., van Gemert, G. J., Bolmer, M. G. \& Meuwissen, J. H. Feeding behaviour and sporozoite ejection by infected Anopheles stephensi. Trans. R. Soc. Trop. Med. Hyg. 85, 175-180 (1991).

25. Medica, D. L. \& Sinnis, P. Quantitative dynamics of Plasmodium yoelii sporozoite transmission by infected anopheline mosquitoes. Infect. Immun. 73, 4363-4369 (2005).

26. Beier, J. C., Davis, J. R., Vaughan, J. A., Noden, B. H. \& Beier, M. S. Quantitation of Plasmodium falciparum sporozoites transmitted in vitro by experimentally infected Anopheles gambiae and Anopheles stephensi. Am. J. Trop. Med. Hyg. 44, 564-570 (1991).

27. Frischknecht, F. et al. Imaging movement of malaria parasites during transmission by Anopheles mosquitoes. Cell. Microbiol. 6, 687-694 (2004).

28. Jin, Y., Kebaier, C. \& Vanderberg, J. Direct microscopic quantification of dynamics of Plasmodium berghei sporozoite transmission from mosquitoes to mice. Infect. Immun. 75, 5532-5539 (2007)

29. Sidjanski, S. \& Vanderberg, J. P. Delayed migration of Plasmodium sporozoites from the mosquito bite site to the blood. Am. J. Trop. Med. Hyg. 57, 426-429 (1997).

Work showing that most sporozoites are deposited into the skin when a mosquito bites.

30. Matsuoka, H., Yoshida, S., Hirai, M. \& Ishii, A. A rodent malaria, Plasmodium berghei, is experimentally transmitted to mice by merely probing of infective mosquito, Anopheles stephensi. Parasitol. Int. 51, 17-23 (2002)

31. Vanderberg, J. P. \& Frevert, U. Intravital microscopy demonstrating antibody-mediated immobilisation of Plasmodium berghei sporozoites injected into skin by mosquitoes. Int. J. Parasitol. 34, 991-996 (2004). The first imaging of sporozoite gliding motility in the skin of mice.

32. Amino, R. et al. Quantitative imaging of Plasmodium transmission from mosquito to mammal. Nature Med. 12, 220-224 (2006).

An article describing the tripartite fate of sporozoites inoculated into the skin.

33. Amino, R. et al. Imaging malaria sporozoites in the dermis of the mammalian host. Nature Protoc. 2 , 1705-1712 (2007)
34. Yamauchi, L. M., Coppi, A., Snounou, G. \& Sinnis, P. Plasmodium sporozoites trickle out of the injection site. Cell. Microbiol. 9, 1215-1222 (2007).

35. Xu, W.-Y., Wang, X.-X., Qi, J., Duan, J.-H. \& Huang, F.-S Plasmodium yoelii: influence of immune modulators on the development of the liver stage. Exp. Parasitol. 126 254-258 (2010)

36. Ellis, J. et al. Cloning and expression in E. coli of the malarial sporozoite surface antigen gene from Plasmodium knowlesi. Nature 302, 536-538 (1983) The first cloning of a Plasmodium spp. gene.

37. Pradel, G., Garapaty, S. \& Frevert, U. Proteoglycans mediate malaria sporozoite targeting to the liver. $\mathrm{Mol}$. Microbiol. 45, 637-651 (2002)

38. Suarez, J. E. et al. Plasmodium falciparum circumsporozoite (CS) protein peptides specifically bind to HepG2 cells. Vaccine 19, 4487-4495 (2001).

39. Rathore, D., Sacci, J. B., de la Vega, P. \& McCutchan, T. F. Binding and invasion of liver cells by Plasmodium falciparum sporozoites. Essential involvement of the amino terminus of circumsporozoite protein. J. Biol. Chem. 277, 7092-7098 (2002)

40. Cerami, C. et al. The basolateral domain of the hepatocyte plasma membrane bears receptors for the circumsporozoite protein of Plasmodium falciparum sporozoites. Cell 70, 1021-1033 (1992).

41. Frevert, U. et al. Malaria circumsporozoite protein binds to heparan sulfate proteoglycans associated with the surface membrane of hepatocytes. J. Exp. Med. 177, 1287-1298 (1993).

42. Sinnis, P. et al. Structural and functional properties of region II-plus of the malaria circumsporozoite protein. J. Exp. Med. 180, 297-306 (1994).

43. Coppi, A. et al. The malaria circumsporozoite protein has two functional domains, each with distinct roles as sporozoites journey from mosquito to mammalian host. J. Exp. Med. 208, 341-356 (2011).

44. Frevert, U. et al. Intravital observation of Plasmodium berghei sporozoite infection of the liver. PLoS Biol. 3, e192 (2005)

45. Mota, M. M., Hafalla, J. C. R. \& Rodriguez, A. Migration through host cells activates Plasmodium sporozoites for infection. Nature Med. 8, 1318-1322 (2002).

46. Carrolo, M. et al. Hepatocyte growth factor and its receptor are required for malaria infection. Nature Med. 9, 1363-1369 (2003).

47. Kaushansky, A.\& Kappe, S. H. I. The crucial role of hepatocyte growth factor receptor during liver-stage infection is not conserved among Plasmodium species. Nature Med. 17, 1180-1181 (2011).

48. Ishino, T., Yano, K., Chinzei, Y. \& Yuda, M. Cell-passage activity is required for the malarial parasite to cross the liver sinusoidal cell layer. PLoS Biol. 2, e4 (2004) The generation of the first Plasmodium spp. sporozoites deficient in CT.

49. Ishino, T., Chinzei, Y. \& Yuda, M. A. Plasmodium sporozoite protein with a membrane attack complex domain is required for breaching the liver sinusoidal cell layer prior to hepatocyte infection. Cell. Microbiol. 7, 199-208 (2005)

50. Yuda, M. \& Ishino, T. Liver invasion by malarial parasites - how do malarial parasites break through the host barrier? Cell. Microbiol. 6, 1119-1125 (2004).

51. Amino, R. et al. Host cell traversal is important for progression of the malaria parasite through the dermis to the liver. Cell Host Microbe 3, 88-96 (2008). 
52. Tavares, J. et al. Role of host cell traversal by the malaria sporozoite during liver infection. J. Exp. Med. 210, 905-915 (2013).

53. Pradel, G. \& Frevert, U. Malaria sporozoites actively enter and pass through rat Kupffer cells prior to hepatocyte invasion. Hepatology 33, 1154-1165 (2001).

54. Baer, K. et al. Kupffer cells are obligatory for Plasmodium yoelii sporozoite infection of the liver. Cell. Microbiol. 9, 397-412 (2007)

55. Coppi, A. et al. Heparan sulfate proteoglycans provide a signal to Plasmodium sporozoites to stop migrating and productively invade host cells. Cell Host Microbe 2, 316-327 (2007)

56. Frevert, U., Sinnis, P., Esko, J. D. \& Nussenzweig, V. Cell surface glycosaminoglycans are not obligatory for Plasmodium berghei sporozoite invasion in vitro. Mol. Biochem. Parasitol. 76, 257-266 (1996).

57. Coppens, I., Sullivan, D. J. \& Prigge, S. T. An update on the rapid advances in malaria parasite cell biology. Trends Parasitol. 26, 305-310 (2010).

58. Spielmann, T., Montagna, G. N., Hecht, L. \& Matuschewski, K. Molecular make-up of the Plasmodium parasitophorous vacuolar membrane. Int. J. Med. Microbiol. 302, 179-186 (2012).

59. Graewe, S., Stanway, R. R., Rennenberg, A. \& Heussler, V. T. Chronicle of a death foretold: Plasmodium liver stage parasites decide on the fate of the host cell. FEMS Microbiol. Rev. 36, 111-130 (2012).

60. Lim, L. \& McFadden, G. I. The evolution, metabolism and functions of the apicoplast. Phil. Trans. R. Soc. 365, 749-763 (2010).

61. Van de Sand, C. et al. The liver stage of Plasmodium berghei inhibits host cell apoptosis. Mol. Microbiol. 58, 731-742 (2005)

62. Rennenberg, A. et al. Exoerythrocytic Plasmodium parasites secrete a cysteine protease inhibitor involved in sporozoite invasion and capable of blocking cell death of host hepatocytes. PLoS Pathog. 6, e1000825 (2010)

63. Kaushansky, A. et al. Suppression of host p53 is critical for Plasmodium liver-stage infection. Cell Rep. 3, 630-637 (2013)

64. Sturm, A. et al. Alteration of the parasite plasma membrane and the parasitophorous vacuole membrane during exo-erythrocytic development of malaria parasites. Protist 160, 51-63 (2009)

65. Schmidt-Christensen, A., Sturm, A., Horstmann, S $\&$ Heussler, V. T. Expression and processing of Plasmodium berghei SERA3 during liver stages. Cell. Microbiol. 10, 1723-1734 (2008).

66. Graewe, S. et al. Hostile takeover by Plasmodium: reorganization of parasite and host cell membranes during liver stage egress. PLoS Pathog. 7, e1002224 (2011).

67. Frevert, U. Sneaking in through the back entrance: the biology of malaria liver stages. Trends Parasitol. 20 417-424 (2004)

68. Sturm, A. et al. Manipulation of host hepatocytes by the malaria parasite for delivery into liver sinusoids. Science 313, 1287-1290 (2006). The first observation of hepatic merozoite release via merosomes.

69. Thiberge, S. et al. In vivo imaging of malaria parasites in the murine liver. Nature Protoc. 2, 1811-1818 (2007).

70. Vaughan, A. M. et al. Complete Plasmodium falciparum liver-stage development in liver-chimeric mice. J. Clin. Invest. 122, 3618-3628 (2012).

71. Baer, K., Klotz, C., Kappe, S. H. I., Schnieder, T. \& Frevert, U. Release of hepatic Plasmodium yoelii merozoites into the pulmonary microvasculature. PLOS Pathog. 3, e171 (2007)

72. Gueirard, P. et al. Development of the malaria parasite in the skin of the mammalian host. Proc. Natl Acad. Sci. USA 107, 18640-18645 (2010) The first observation of merozoite formation in the skin of mice.

73. Voza, T., Miller, J. L., Kappe, S. H. I. \& Sinnis, P. Extrahepatic exoerythrocytic forms of rodent malaria parasites at the site of inoculation: clearance after immunization, susceptibility to primaquine, and contribution to blood-stage infection. Infect. Immun 80, 2158-2164 (2012).

74. Mellor, A. L. \& Munn, D. H. Immune privilege: a recurrent theme in immunoregulation? Immunol. Rev. 213, 5-11 (2006)

75. Frevert, U., Späth, G. F. \& Yee, H. Exoerythrocytic development of Plasmodium gallinaceum in the White Leghorn chicken. Int. J. Parasitol. 38, 655-672 (2008).
76. Silvie, O. et al. Hepatocyte CD81 is required for Plasmodium falciparum and Plasmodium yoelii sporozoite infectivity. Nature Med. 9, 93-96 (2003)

77. Boyd, M. F. \& Kitchen, S. F. The demonstration of sporozoites in human tissues. Am. J. Trop. Med. Hyg. 19, 27-31 (1939)

78. Offeddu, V., Thathy, V., Marsh, K. \& Matuschewski, K. Naturally acquired immune responses against Plasmodium falciparum sporozoites and liver infection. Int. J. Parasitol. 42, 535-548 (2012).

79. Hoffman, S. L. et al. Protection of humans against malaria by immunization with radiation-attenuated Plasmodium falciparum sporozoites. J. Infect. Dis. 185, 1155-1164 (2002)

80. Mueller, A.-K., Labaied, M., Kappe, S. H. I. \& Matuschewski, K. Genetically modified Plasmodium parasites as a protective experimental malaria vaccine. Nature 433, 164-167 (2005) The first GAP-induced protection against sporozoite challenge in mice.

81. Mueller, A.-K et al. Plasmodium liver stage developmental arrest by depletion of a protein at the parasite-host interface. Proc. Natl Acad. Sci. USA 102 3022-3027 (2005)

82. van Dijk, M. R. et al. Genetically attenuated, 36p-deficient malarial sporozoites induce protective immunity and apoptosis of infected liver cells. Proc Natl Acad. Sci. USA 102, 12194-12199 (2005).

83. Labaied, M. et al. Plasmodium yoelii sporozoites with simultaneous deletion of P52 and P36 are completely attenuated and confer sterile immunity against infection. Infect. Immun. 75, 3758-3768 (2007).

84. Jobe, O. et al. Genetically attenuated Plasmodium berghei liver stages induce sterile protracted protection that is mediated by major histocompatibility complex class I-dependent interferon- $\gamma$-producing CD8 ${ }^{+}$T cells. J. Infect. Dis. 196, 599-607 (2007)

85. Mueller, A.-K. et al. Genetically attenuated Plasmodium berghei liver stages persist and elicit sterile protection primarily via CD8 T cells. Am. J. Pathol. 171, 107-115 (2007)

86. Tarun, A. S. et al. Protracted sterile protection with Plasmodium yoelii pre-erythrocytic genetically attenuated parasite malaria vaccines is independent of significant liver-stage persistence and is mediated by CD8 ${ }^{+} \mathrm{T}$ cells. J. Infect. Dis. 196, 608-616 (2007).

87. Vaughan, A. M. et al. Type II fatty acid synthesis is essential only for malaria parasite late liver stage development. Cell. Microbiol. 11, 506-520 (2009).

88. Pei, Y. et al. Plasmodium pyruvate dehydrogenase activity is only essential for the parasite's progression from liver infection to blood infection. Mol. Microbiol. 75, 957-971 (2010)

89. Haussig, J. M., Matuschewski, K. \& Kooij, T. W. A Inactivation of a Plasmodium apicoplast protein attenuates formation of liver merozoites. Mol. Microbiol. 81, 1511-1525 (2011)

90. Butler, N. S. et al. Superior antimalarial immunity after vaccination with late liver stage-arresting genetically attenuated parasites. Cell Host Microbe 9 , 451-462 (2011).

Work showing that late-arrested liver-stage GAP protect better than RAS.

91. Falae, A. et al. Role of Plasmodium berghei cGMP dependent protein kinase in late liver stage development. J. Biol. Chem. 285, 3282-3288 (2010).

92. Beaudoin, R. L., Strome, C. P. A., Mitchell, F. \& Tubergen, T. A. Plasmodium berghei: immunization of mice against the ANKA strain using the unaltered sporozoite as an antigen. Exp. Parasitol. 42, 1-5 (1977).

The first use of DAP to protect against malaria in rodents.

93. Orjih, A. U., Cochrane, A. H. \& Nussenzweig, R. S. Comparative studies on the immunogenicity of infective and attenuated sporozoites of Plasmodium berghei. Trans. R. Soc. Trop. Med. Hyg. 76, 57-61 (1982)

94. Belnoue, E. et al. Protective T cell immunity against malaria liver stage after vaccination with live sporozoites under chloroquine treatment. J. Immunol. 172, 2487-2495 (2004).

95. Inoue, M. \& Culleton, R. L. The intradermal route for inoculation of sporozoites of rodent malaria parasites for immunological studies. Parasite Immunol. 33, 137-142 (2011).

96. Friesen, F. et al. Natural immunization against malaria: causal prophylaxis with antibiotics. Sci. Transl. Med. 14, 40 ra49 (2010).
97. Friesen, J. \& Matuschewski, K. Comparative efficacy of pre-erythrocytic whole organism vaccine strategies against the malaria parasite. Vaccine 29, 7002-7008 (2011).

98. Roestenberg, M. et al. Protection against a malaria challenge by sporozoite inoculation. N. Engl. J. Med. 361, 468-477 (2009).

\section{Validation of the DAP concept in humans.}

99. Roestenberg, M. et al. Long-term protection against malaria after experimental sporozoite inoculation: an open-label follow-up study. Lancet 377, 1770-1776 (2011).

100. Purcell, L. A. et al. Chemically attenuated Plasmodium sporozoites induce specific immune responses, sterile immunity and cross-protection against heterologous challenge. Vaccine 26, 4880-4884 (2008).

101. Ngonseu, E., Chatterjee, S. \& Wery, M. Blocked hepatic-stage parasites and decreased susceptibility to Plasmodium berghei infections in BALB/c mice. Parasitology 117, 419-423 (1998)

102. Schmidt, N. W., Butler, N. S. \& Harty, J. T. Plasmodium-host interactions directly influence the threshold of memory CD8 T cells required for protective immunity. J. Immunol. 186, 5873-5884 (2011)

103. Yoshida, N., Nussenzweig, R. S., Potocnjak, P., Nussenzweig, V. \& Aikawa, M. Hybridoma produces protective antibodies directed against the sporozoite stage of malaria parasite. Science 207, 71-73 (1980).

The first demonstration that antibodies to CSP neutralize infectivity.

104. Stewart, M. J., Nawrot, R. J., Schulman, S. \& Vanderberg, J. P. Plasmodium berghei sporozoite invasion is blocked in vitro by sporozoite-immobilizing antibodies. Infect. Immun. 51, 859-864 (1986).

105. Schofield, L. et al. $\gamma$-interferon, CD8 ${ }^{+} \mathrm{T}$ cells and antibodies required for immunity to malaria sporozoites. Nature 330, 664-666 (1987). The first evidence that $\mathrm{CD}^{+} \mathrm{T}$ cells are important for RAS-induced protection in mice.

106. Weiss, W. R., Sedegah, M., Beaudoin, R. L., Miller, L. H. \& Good, M. F. CD8 ${ }^{+}$T cells (cytotoxic/suppressors) are required for protection in mice immunized with malaria sporozoites. Proc. Natl Acad. Sci. USA 85, 573-576 (1988)

107. Romero, P. et al. Cloned cytotoxic T cells recognize an epitope in the circumsporozoite protein and protect against malaria. Nature 341, 323-326 (1989).

108. Rodrigues, M. M. et al. CD8 ${ }^{+}$cytolytic T cell clones derived against the Plasmodium yoelii circumsporozoite protein protect against malaria. Int. Immunol. 3, 579-585 (1991)

109. Tsuji, M., Romero, P., Nussenzweig, R. S. \& Zavala, F. $\mathrm{CD} 4^{+}$cytolytic $\mathrm{T}$ cell clone confers protection against murine malaria. J. Exp. Med. 172, 1353-1357 (1990).

110. Rénia, L. et al. Effector functions of circumsporozoite peptide-primed $\mathrm{CD} 4^{+} \mathrm{T}$ cell clones against Plasmodium yoelii liver stages. J. Immunol. 150 1471-1478 (1993).

111. Chakravarty, S., Baldeviano, G. C., Overstreet, M. G. \& Zavala, F. Effector CD8 ${ }^{+}$T lymphocytes against liver stages of Plasmodium yoelii do not require $\gamma$ interferon for antiparasite activity. Infect. Immun. 76, 3628-3631 (2008).

112. Butler, N. S., Schmidt, N. W. \& Harty, J. T. Differential effector pathways regulate memory CD8 T cell immunity against Plasmodium berghei versus $P$. yoeli sporozoites. J. Immunol. 184, 2528-2538 (2010).

113. Cockburn, I. A. et al. In vivo imaging of $\mathrm{CD}^{+}{ }^{+} \mathrm{T}$ cellmediated elimination of malaria liver stages. Proc. Natl Acad. Sci. USA 110, 9090-9095 (2013). The first In vivo imaging of pathogen killing by CD8 T cells.

114. Cohen, J., Nussenzweig, V., Nussenzweig, R., Vekemans, J. \& Leach, A. From the circumsporozoite protein to the RTS, S/AS candidate vaccine. Hum. Vaccines 6, 90-96 (2010).

115. Luke, T. C. \& Hoffman, S. L. Rationale and plans for developing a non-replicating, metabolically active, radiation-attenuated Plasmodium falciparum sporozoite vaccine. J. Exp. Biol. 206, 3803-3808 (2003).

116. Epstein, J. E. et al. Live attenuated malaria vaccine designed to protect through hepatic $\mathrm{CD} 8{ }^{+} \mathrm{T}$ cell immunity. Science 334, 475-480 (2011).

117. Nganou-Makamdop, K. \& Sauerwein, R. W. Liver or blood-stage arrest during malaria sporozoite immunization: the later the better? Trends Parasitol. 29, 304-310 (2013). 
118. Stoute, J. A. et al. A preliminary evaluation of a recombinant circumsporozoite protein vaccine against Plasmodium falciparum malaria. N. Engl. J. Med. 336, 86-91 (1997).

119. Kester, K. E. et al. Phase 2 a trial of 0,1 , and 3 month and 0,7 , and 28 day immunization schedules of malaria vaccine RTS,S/ASO2 in malaria-naïve adults at the Walter Reed Army Institute of Research. Vaccine 26, 2191-2202 (2008).

120. Kester, K. E. et al. Randomized, double-blind, phase 2a trial of falciparum malaria vaccines RTS,S/ASO1B and RTS, S/AS02A in malaria-naive adults: safety, efficacy, and immunologic associates of protection. J. Infect. Dis. 200, 337-346 (2009).

121. Agnandji, S. T. et al. First results of phase 3 trial of RTS,S/AS01 malaria vaccine in African children. N. Engl. J. Med. 365, 1863-1875 (2011)

122. Nussenzweig, V., Good, M. F. \& Hill, A. V. S. Mixed results for a malaria vaccine. Nature Med. 17 1560-1561 (2011).

123. Olotu, A. et al. Four-year efficacy of RTS, S/AS01E and its interaction with malaria exposure. N. Engl. J. Med. 368, 1111-1120 (2013).

124. Mellouk, S., Lunel, F., Sedegah, M., Beaudoin, R. \& Druilhe, P. Protection against malaria induced by irradiated sporozoites. Lancet 335, 721 (1990)

125. Chatterjee, S., François, G., Druilhe, P., Timperman, G. ¿ Wéry, M. Immunity to Plasmodium berghei exoerythrocytic forms derived from irradiated sporozoites. Parasitol. Res. 82, 297-303 (1996).

126. Silvie, O. et al. Effects of irradiation on Plasmodium falciparum sporozoite hepatic development: implications for the design of pre-erythrocytic malaria vaccines. Parasite Immunol. 24, 221-223 (2002).

127. Pombo, D. J. et al. Immunity to malaria after administration of ultra-low doses of red cells infected with Plasmodium falciparum. Lancet 360, 610-617 (2002).

128. Bijker, E. M. et al. Protection against malaria after immunization by chloroquine prophylaxis and sporozoites is mediated by preerythrocytic immunity. Proc. Natl Acad. Sci. USA 110, 7862-7867 (2013).

129. Yu, M. et al. The fatty acid biosynthesis enzyme Fabl plays a key role in the development of liver-stage malarial parasites. Cell Host Microbe 4, 567-578 (2008).

Work showing an essential role for the parasite apicoplast in the late phase of liver-stage development.
130. Lasonder, E et al. Proteomic profiling of Plasmodium sporozoite maturation identifies new proteins essential for parasite development and infectivity. PLoS Pathog. 4, e1000195 (2008)

131. Tarun, A. S. et al. A combined transcriptome and proteome survey of malaria parasite liver stages. Proc. Natl Acad. Sci. USA 105, 305-310 (2008).

132. Chakravarty, S. et al. CD8 ${ }^{+}$T lymphocytes protective against malaria liver stages are primed in skin-draining lymph nodes. Nature Med. 13, 1035-1041 (2007). A study which finds that protective immune responses to sporozoites are generated in skin-draining lymph nodes.

133. Obeid, M. et al. Skin-draining lymph node priming is sufficient to induce sterile immunity against preerythrocytic malaria. EMBO Mol. Med. 5, 250-263 (2013).

134. Sano, G. et al. Swift development of protective effector functions in naive $C D 8^{+} \mathrm{T}$ cells against malaria liver stages. J. Exp. Med. 194, 173-179 (2001).

135. Jung, S. et al. In vivo depletion of $\mathrm{CD} 11 \mathrm{c}^{+}$dendritic cells abrogates priming of $C D 8^{+} \mathrm{T}$ cells by exogenous cellassociated antigens. Immunity 17, 211-220 (2002).

136. Cockburn, I. A. et al. Dendritic cells and hepatocytes use distinct pathways to process protective antigen from Plasmodium in vivo. PLoS Pathog. 7, e 1001318 (2011). The PEXEL motif is shown to be dispensable for presentation of the CSP antigen.

137. Cockburn, I. A. et al. Prolonged antigen presentation is required for optimal $C D 8^{+} \mathrm{T}$ cell responses against malaria liver stage parasites. PLoS Pathog. 6 , e1000877 (2010)

138. Hafalla, J. C. R. et al. Priming of $\mathrm{CD}^{+} \mathrm{T}$ cell responses following immunization with heat-killed Plasmodium sporozoites. Eur. J. Immunol. 36, 1179-1186 (2006).

139. Seder, R. A. et al. Protection against malaria by intravenous immunization with a nonreplicating sporozoite vaccine. Science http://dx.doi.org/10.1126/ science. 1241800 (2013).

The first demonstrated protection of humans by parenteral injection of RAS.

140. Nganou-Makamdop, K. et al. Reduced Plasmodium berghei sporozoite liver load associates with low protective efficacy after intradermal immunization. Parasite Immunol. 34, 562-569 (2012).

141. Voza, T., Kebaier, C. \& Vanderberg, J. P. Intradermal immunization of mice with radiation-attenuated sporozoites of Plasmodium yoelii induces effective protective immunity. Malaria J. 9, 362 (2010).
142. Ploemen, I. H. et al. Plasmodium liver load following parenteral sporozoite administration in rodents. Vaccine 31, 3410-3416 (2013).

143. Bongfen, S. E., Torgler, R., Romero, J. F., Rénia, L. \& Corradin, G. Plasmodium berghei-infected primary hepatocytes process and present the circumsporozoite protein to specific $\mathrm{CD}^{+}{ }^{+} \mathrm{T}$ cells in vitro. J. Immunol. 178, 7054-7063 (2007)

144. Spielmann, T. \& Gilberger, T.-W. Protein export in malaria parasites: do multiple export motifs add up to multiple export pathways? Trends Parasitol. 26, 6-10 (2010)

145. Grüring, C. et al. Uncovering common principles in protein export of malaria parasites. Cell Host Microbe 12, 717-729 (2012)

146. Singh, A. P. et al. Plasmodium circumsporozoite protein promotes the development of the liver stages of the parasite. Cell 131, 492-504 (2007).

147. Ginhoux, F., Ng, L. G. \& Merad, M. Understanding the murine cutaneous dendritic cell network to improve intradermal vaccination strategies. Curr. Top. Microbiol. Immunol. 351, 1-24 (2012).

148. Guilbride, D. L., Gawlinski, P. \& Guilbride, P. D. L. Why functional pre-erythrocytic and bloodstage malaria vaccines fail: a meta-analysis of fully protective immunizations and novel immunological model. PLoS ONE 5, e 10685 (2010).

149. Druilhe, P. \& Barnwell, J. W. Pre-erythrocytic stage malaria vaccines: time for a change in path. Curr. Opin. Microbiol. 10, 371-378 (2007).

150. Hill, A. V. S. et al. Prime-boost vectored malaria vaccines: progress and prospects. Hum. Vaccines 6 78-83 (2010).

151. Reyes-Sandoval, A. et al. CD8 ${ }^{+}$T effector memory cells protect against liver-stage malaria. J. Immunol. 187, 1347-1357 (2011)

152. Kumar, K. A. et al. The circumsporozoite protein is an immunodominant protective antigen in irradiated sporozoites. Nature 444, 937-940 (2006).

153. Mauduit, M. et al. Minimal role for the circumsporozoite protein in the induction of sterile immunity by vaccination with live rodent malaria sporozoites. Infect. Immun. 78, 2182-2188 (2010).

154. Murphy, S. C., Kas, A., Stone, B. C. \& Bevan, M. J. A T-cell response to a liver-stage Plasmodium antigen is not boosted by repeated sporozoite immunizations Proc. Natl Acad. Sci. USA 110, 6055-6060 (2013).

Competing interests statement

The authors declare no competing financial interests. 


\section{CORRIGENDUM}

Looking under the skin: the first steps in malarial infection and immunity

Robert Ménard, Joana Tavares, Ian Cockburn, Miles Markus, Fidel Zavala and Rogerio Amino

Nature Reviews Microbiology 11, 701-712 (2013)

In Box 3 of the above article (page 709) the sentence "Recently, it was found that five intravenous injections of $>10^{5}$ cryopreserved RAS protected all six tested patients against a challenge by bites of five mosquitoes 3 weeks after the last immunization dose, whereas four intravenous immunizations protected only three of nine patients ${ }^{139}$ " should have read

"Recently, it was found that five intravenous injections of $>10^{5}$ cryopreserved RAS protected all six tested patients against a challenge by bites of five mosquitoes 3 weeks after the last immunization dose, whereas four intravenous immunizations protected only six of nine patients ${ }^{139}$." The authors apologize to readers for any misunderstanding caused. This has now been corrected online. 